\title{
Research methods of a heated space temperature adjustment system with respect to endogenous and exogenous uncertainties
}

\author{
Nikolai Tseligorov ${ }^{1,}{ }^{*}$, Anatoly Tchubukin ${ }^{1}$, Anatoly Ozersky ${ }^{1}$, Ahmed Naser $^{1}$, and Elena \\ Tseligorova $^{1}$ \\ ${ }^{1}$ Don state technical university, 344003, 1, Gagarin square, Rostov-on-Don, Russia
}

\begin{abstract}
Research stages of the indoor temperature adjustment system have been suggested. The capability assessment of indoor temperature adjustment with analog and discrete models of the control system was carried out. Robust absolute stability of the control system to the influence of endogenous and exogenous uncertainties was studied. Analytical results of the investigation were confirmed by a simulation conducted with the help of Matlab
\end{abstract}

\section{Introduction}

To keep the air temperature indoors, the heating systems are used that depending on exterior temperature, a heater capacity, admission of sunlight, windspeed outdoors, and other parameters maintain the required temperature conditions. The heating system consists of three basic elements: a source of heat, piping systems, heating equipment, and a regulator. At the majority of heat boiler stations different analog regulators are used, they have well known drawbacks which can be significantly reduced by means of introducing digital controllers at contemporary independent heat boiler stations; these regulators are able to respond to weather changes in a few minutes, and this allows to save power [1, 2]. Besides, a digital control system makes it possible to control indoor temperature more precisely compared to analog/thermostat system where heating inertia can cause about $1.5^{\circ}$ $\mathrm{C}$ indoor temperature variations resulting in thermal discomfort [3]. Modern systems of indoor temperature automatic control are the systems which function despite the availability of uncertainties of many types [4-7]. To take into account concomitant nonlinearities and other factors in mathematical models of temperature control systems which refer to nonlinear discrete automatic control systems (NDACS), it is necessary to apply special methods of research that could take into account not only structural peculiarities of NDACS, but also a model stability to uncertainties (robustness).

\section{The problem statement}

Corresponding author: nzelig@rambler.ru. 
At present, there are a lot of published papers relating to mathematical simulation of temperature conditions of buildings corresponding to residential, livestock, and industrial buildings. In the papers [8-10] mathematical models of temperature adjusting systems inside residential buildings are considered. Insolation of buildings is considered in the papers $[10,11]$.

Transfer function of a heated space in these papers is written as:

$$
W(p)=\frac{K}{T_{b} p+1}
$$

where $K$ is a function of the following thermophysical quantities:

$Q$ - thermal energy coming from a heater;

$k_{o k}$ - heat transmission coefficient of building envelopes (walls);

$F_{o k}$ - surface areas of building envelopes (walls);

$t_{b}$ - temperature of building envelopes (walls);

$t_{\text {in }}$ - room temperature.

On the other hand, time constant of the heated room $T_{b}$ also depends on the following quantities:

$G_{b}$ - room mass;

$k_{o k}$ - heat transmission coefficient of building envelopes (walls);

$F_{o k}$ - surface areas of building envelopes (walls);

$c_{b}$ - specific thermal capacity.

The analysis of the transfer function $W(p)$ and the time constant $T_{b}$ of the heated space indicates that the system under consideration contains the following uncertainties $[6,7]$ :

a) exogenous (depends on exterior temperature);

b) endogenous (depends on the supplied thermal energy and the heated space mass).

The problem to be set is to study the heating system as a discrete one taking into account influence of temperature and other uncertainties on robust stability. The heating system simulation is to be carried out with the help of the software product Matlab/ Simulink.

\section{The problem solution}

In order to solve the formulated problem, it is necessary to carry out synthesis and simulation of the heating system in a sequential order starting to consider it as an analog system, and then as a discrete one. At the next stage it is necessary to make study of the heating system taking into consideration the basic uncertainties available in the system, i.e. to study the system with interval coefficients of the transfer function.

\subsection{Study of the system analog model}

Let us make a synthesis of the regulator and study of the room temperature change control system as an analog model depending on exogenous uncertainties.

To perform simulation, we use the following transfer functions $[12,13]$ of:

- a heated space

$$
W(s)=\frac{0,78}{502 s+1}
$$


- a temperature sensor

$$
W_{d}(s)=\frac{1}{20 s+1}
$$

To perform synthesis of analog PI-controller we use the software program pidtune of the system MATLAB with the help of which we determine parameters of the controller: $\mathrm{Kp}=4,618, \mathrm{Ki}=0,0095$. A structural diagram of the analog system under Matlab is presented in fig.1.

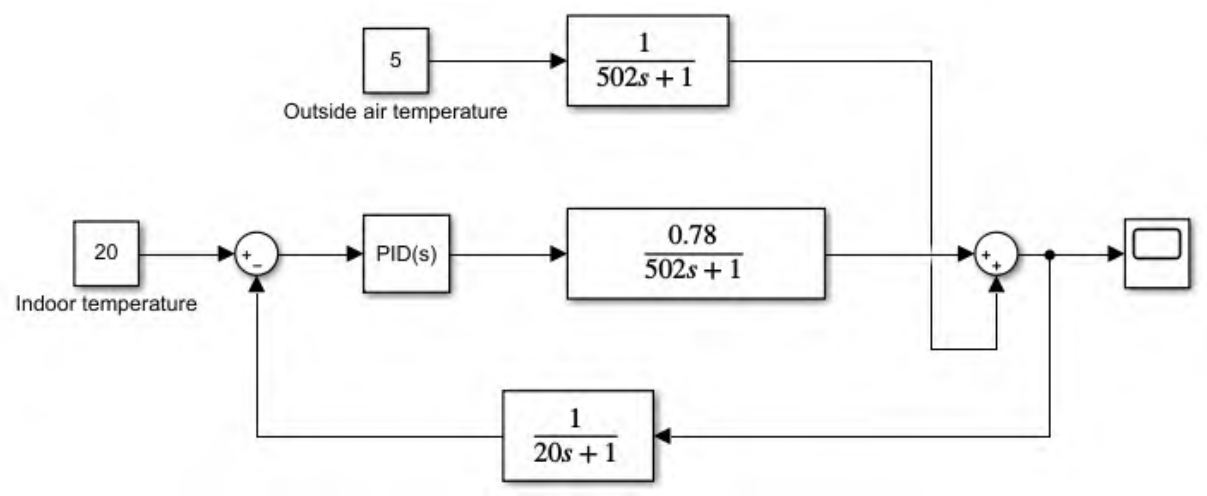

Fig. 1. Structural diagram of the closed system of temperature control

Let us plot a chart of the heated space temperature change depending on the outdoor temperature $t_{o}=5^{0} \mathrm{C}$ (fig.2).

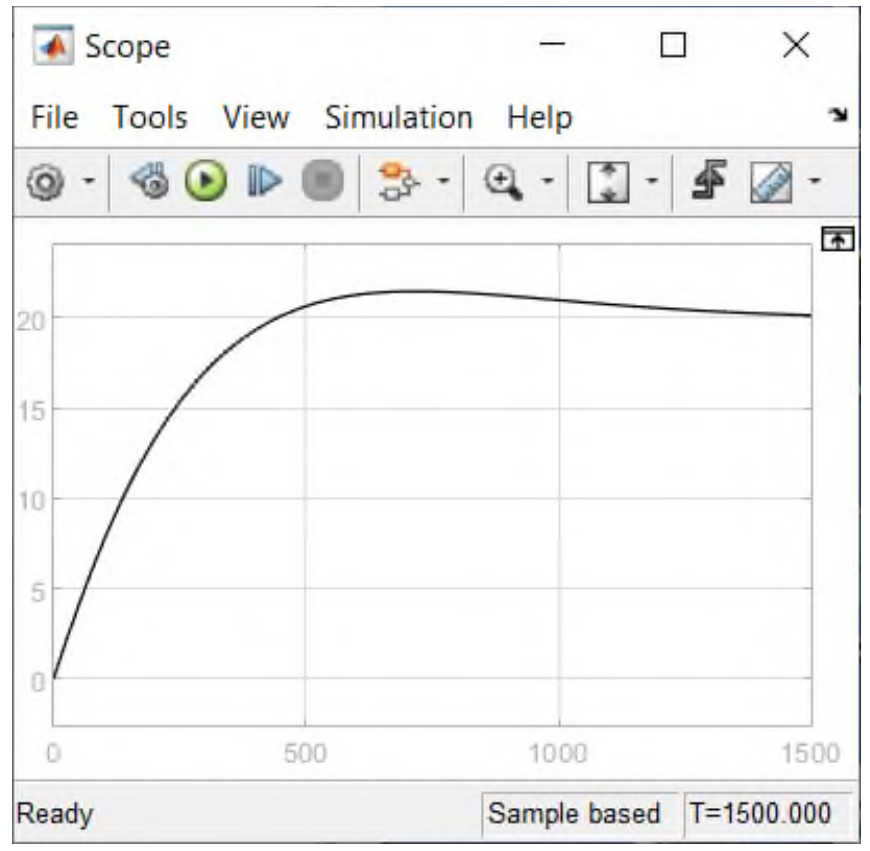

Fig. 2. Temperature curve of the heated space 
In the presented curve we see that indoor temperature is set at a pregiven level after the transient period have died out with slight readjustment during $1300 \mathrm{~s}$. However, analog regulator does not possess stability of the design parameters and so does not provide the necessary level of robustness under the conditions of possible parameters change of the controllable object.

To eliminate this drawback, we design discrete system of temperature control and study it for the conditions of the parameters pregiven change of the object dynamic model.

\subsection{Synthesis and study of the system discrete model}

With a view to achieve accuracy of the design parameters of the digital temperature controller, we take advantage of the object digital model having obtained it with the help of the function c2d with respect to zero order hold device and a sampling period $\mathrm{T}=1 \mathrm{sec}$. Upon that, we perform synthesis of the digital PID-controller with the help of the program pidtool and the obtained digital model of the object. Herewith, as a preliminary criterion for achieving robustness of the system being designed we use maximum closed-loop frequency response of the system, the less it is, the bigger change of the object parameters can be admitted without loss of stability. The controller sampled data transfer function obtained as a result of the synthesis has the form:

$$
D(z)=\frac{9,650 z^{2}-0,522 z+3,907}{1,667 z^{2}-2,333 z+1} .
$$

A structural diagram, made for simulating in Matlab, is presented in fig.3.

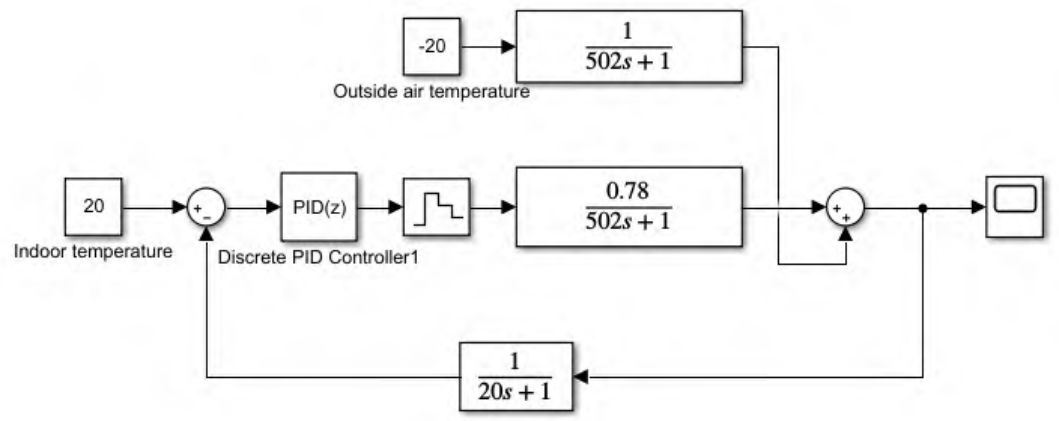

Fig. 3. A structural diagram of the closed loop temperature control system with a controller

Temperature curve of the heated space depending on the outside air temperature $t_{o}=-20^{0}$ is presented in fig. 4 . 


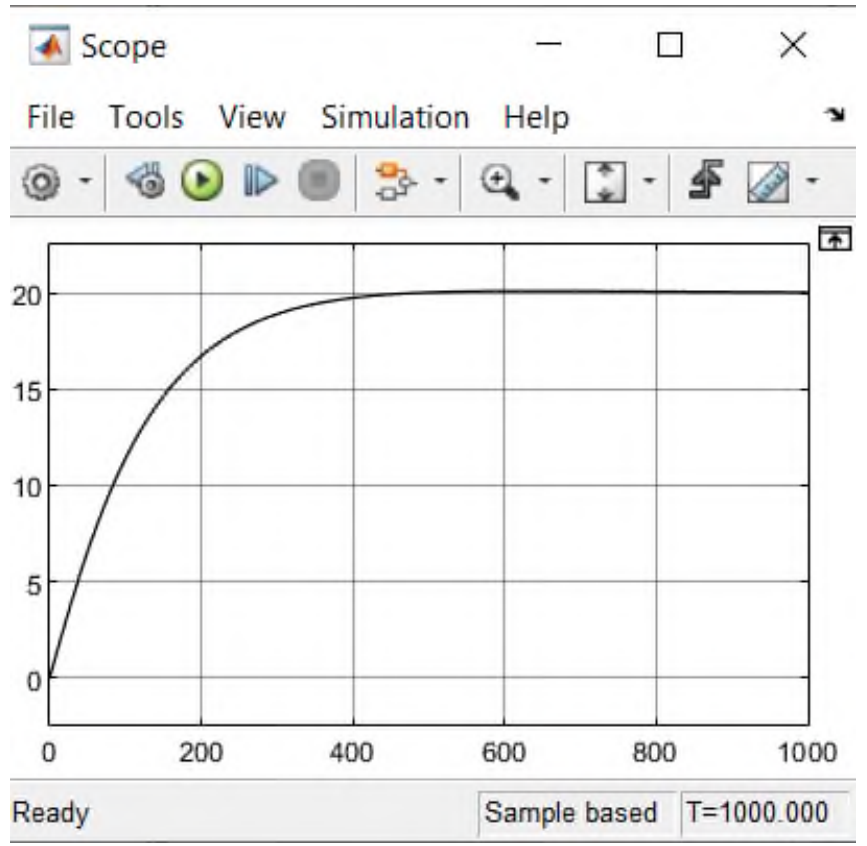

Fig. 4. Temperature curve of the heated space

From the presented curve we can see that a discrete system with a controller provides indoor temperature maintenance in accordance with the task.

\subsection{Assessment of the robust stability of the heated space temperature control system}

At present, a great number of published papers are devoted to the study of robust stability of control systems. These studies are carried on by means of both analytical $[14,15]$ and graphical analytic methods $[17,18]$.

Graphical analytic method of robust absolute stability investigation applied in this paper is based on the combined application of the results, obtained by Y.Z. Tsipkin and V.L. Kharitonov.

When using the analog of Y.Z. Tsipkin criterion after the w-transformation and transition to the pseudofrequency $j v$, we get the following criterion which will be written in the form [17]

$$
R e\left[\left(1+q \frac{2 j v}{1+j v}\right) W(j v)\right]+\frac{1}{k}>0, \quad \forall v \in[0, \infty]
$$

Graphically the given criterion represents a modified amplitude-phase characteristic drawn in the plane $\widetilde{W}^{*}(j v)$. Just here, separated by a dot $1 / \mathrm{k}$ we draw a Popov straight line with a slope of 1/q. Figure 5 shows a graphic illustration of the criterion of absolute stability. 


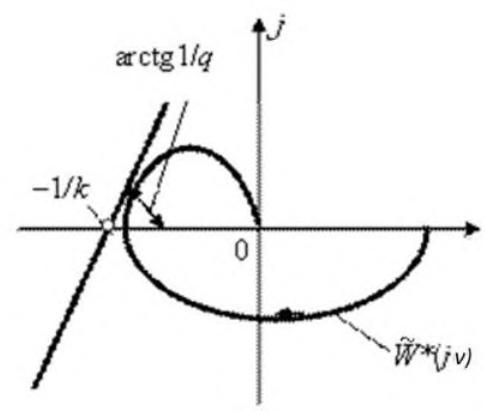

Fig.5. Graphic illustration of the criterion of absolute stability

If $W(j v)$ from (4) is presented in the form

$$
W(j v)=\frac{\alpha_{1}(v)+j \beta_{1}(v)}{\alpha_{2}(v)+j \beta_{2}(v)}
$$

then (4) can be presented by the following polynomial integrand

$$
\begin{aligned}
& k\left\{\left(\alpha_{1}(v) \alpha_{2}(v)+\beta_{1}(v) \beta_{2}(v)\right)\left(1+v^{2}\right)+2 q\left[\left(\alpha_{1}(v) \alpha_{2}(v)+\beta_{1}(v) \beta_{2}(v)\right) v^{2}+\right.\right. \\
& \left.\left.+\left(\alpha_{1}(v) \beta_{2}(v)-\alpha_{2}(v) \beta_{1}(v)\right) v\right]\right\}+\left[\alpha_{2}^{2}(v)+\beta_{2}^{2}(v)\right]\left(1+v^{2}\right)=0 .
\end{aligned}
$$

Then we use the results of the strong theorem by V.L. Kharitonov [6]. As it follows from this theorem, a necessary and sufficient condition of the robust stability of the family of polynomials is to meet the Hurwitz criterion in the following four polynomials:

$$
\begin{aligned}
& P_{1}(x)=\underline{a_{0}}+\bar{a}_{1} x+\bar{a}_{1} x^{2}+a_{3} x^{3}+a_{4} x^{4}+\cdots \\
& P_{2}(x)=\underline{a_{0}}+a_{1} x+\bar{a}_{2} x^{2}+\overline{\bar{a}}_{3} x^{3}+\bar{a}_{4} x^{4}+\cdots \\
& P_{3}(x)=\overline{\bar{a}}_{0}+\overline{\bar{a}}_{1} x+\underline{a_{2}} x^{2}+a_{3} x^{3}+\overline{\bar{a}}_{4} x^{4}+\cdots \\
& P_{3}(x)=\bar{a}_{0}+\underline{a_{1}} x+\underline{a_{2}} x^{2}+\overline{\bar{a}}_{3} x^{3}+\bar{a}_{4} x^{4}+\cdots
\end{aligned}
$$

Substituting coefficients with interval values, obtained from (5) according to the indicated schematic representation in (6), a study of the system for robust absolute stability is carried on using construction of the modified amplitude-phase characteristic for each polynomial $P_{i}$. Therefore, if the obtained amplitude-phase characteristics do not cross Popov straight line, the discrete system under investigation is absolutely robust stable.

Let us make performance assessment of the indoor temperature control system when the values of the transfer function coefficients are changed within the limits of $\pm 10 \%$. These changes can occur in the control system due to ageing of the device components, because of inaccurate tolerances, measurements, errors, and other factors.

On the basis of the above mentioned, transfer function coefficients of the controlled object take the following interval values:

$$
W(s)=\frac{(14,04 . .17,16) s+(0,702 . .0 .858)}{(34486,2 . .42149,8) s^{2}+(440,1 . .537,8) s+(0.9 . .1,1)} .
$$

Our task is to carry on assessment of the robust stability of the sampled-data system with a transfer function, a linear continuous part of which is written in the form (7).

The transfer function of the linear continuous part with interval coefficients has the following form after $\mathrm{w}$-transformation: 


$$
W(w)=\frac{-(1,84 E-07 . .1,52 E-07) w^{2}-(4,23 E-05 . .3,46 E-05) w+(6,99 E-05 . .8,54 E-05)}{(0,9 . .1,1) w^{2}+(0,0468 . .0,0572) w+(8.96 E-05 . .1,09 E-04)} .
$$

Now, let us check robust absolute stability of the obtained control system using graphical method [14], under the condition when $\mathrm{k}=1$ and $\mathrm{q}=1$.

After the w-transformation of the PID controller transfer function, we get the following transfer function:

$$
D(w)=\frac{2,816 w^{2}+4,595 w+10,430}{w^{2}+0,533 w+0,267}
$$

Taking into account (9), the corrected transfer function of NDACS now has the following form:

$$
\begin{gathered}
W(w)=\frac{-(5,20 E-07 . .4,26 E-07) w^{4}-(1.19 E-04 . .9 .9 E-05) w^{3}+(3,605 E-05 . .4,406 E-05) w^{2}-}{(0,9 . .1,1) w^{4}+(0,524 . .0,644) w^{3}+(0,265 . .0,324) w^{2}+} \\
\frac{-(4,903 E-05 . .4,013 E-05) w+(7,30 E-04 . .8,90 E-04)}{+(0,0125 . .0,0153) w+(2,372 E-05 . .2,898 E-05)}
\end{gathered}
$$

Let us check the robust absolute stability of the obtained control system (7) applying graphical method $[14,17]$.

Fig.6 shows the modified amplitude-phase characteristics of the control system.

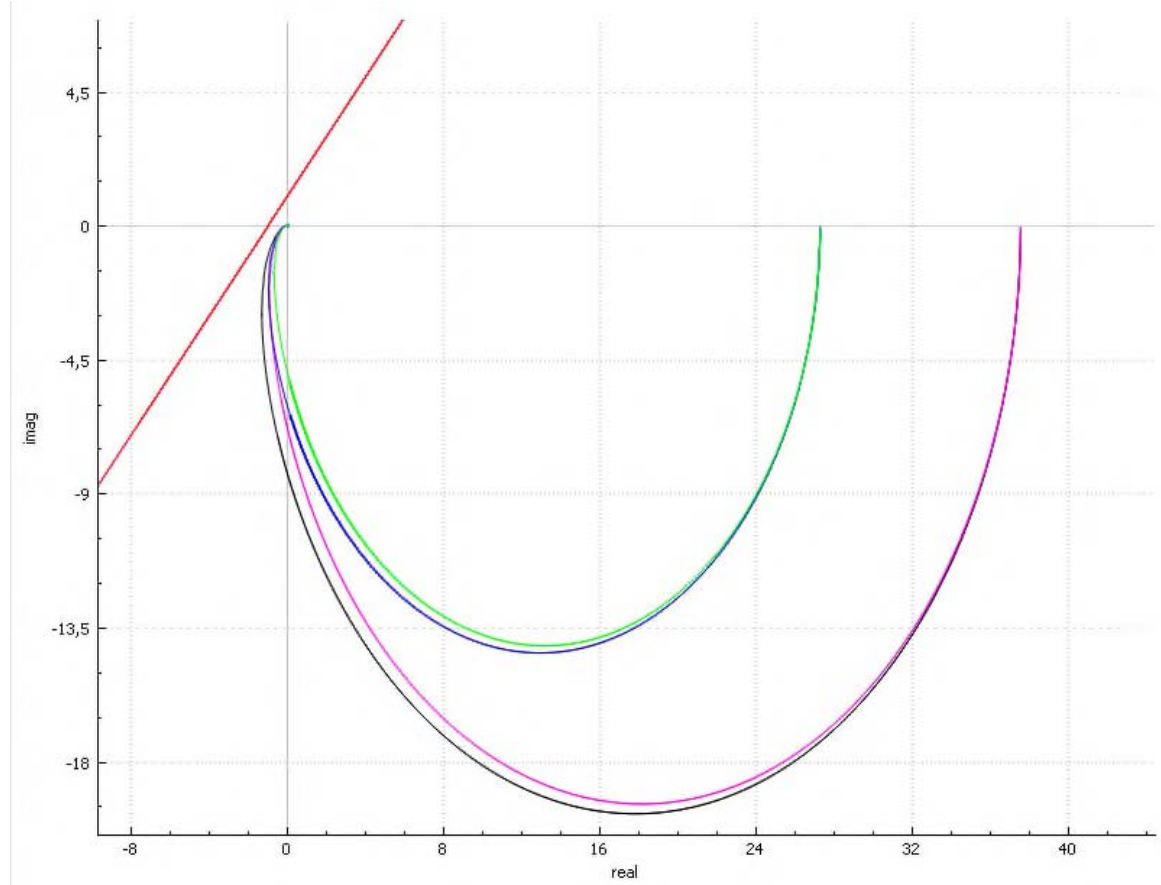

Fig. 6. The modified amplitude-phase characteristics of the compensated control system of indoor temperature

From fig. 6 we see that our control system possesses absolute robust stability as all amplitude-phase characteristics are on the right of Popov line. 


\section{Conclusion}

The advantages of the discrete model of the temperature control system compared to the analog one, consisting in more accurate temperature control, and also the possibility to apply robust approach to the study of the given system were analyzed. It was found out that possible deviations of numerical values of the coefficients of the discrete transfer function of the controlled object within the limits of $\pm 10 \%$ of the reference value do not influence the robust absolute stability of the control system being studied.

\section{References}

1. T. Wellem, B. A. Setiawan, International Journal of Computer Applications, 53, 710(2012)

2. J. Dong, Procedia Computer Science, 107, 196-201 (2017) doi: 10.1016/j.procs.2017.03.078

3. S. Takada, S. Matsumoto, T. Matsushita, Building and Environment, 68, 123-133 (2013)

4. W. Widhiada, D. Negara, P. Suryawan, ATMega, 19(20), 1748-1751 (2017)

5. S. Raju, G. Sinha, Advances in Intelligent Systems and Computing, 1090, 219-226 (2020)

6. N. A. Tseligorov, E. N. Tseligorova, G. M. Mafura, 4-2, 106-111 (2012) http://www.ivdon.ru/magazine/archive/N4t2y2012/ 1340 (Last accessed 11.07.2020)

7. Z. O’Neill, B. Eisenhower, Building Simulation, 6(4), 365-377 (2013)

8. A. Sedov, A. Ainagulova, A. Temirgaliyeva, 3rd International Conference on Mechatronics, Robotics and Automation (2015) doi:10.2991/icmra-15.2015.206

9. M. Georgescu, I. Mezic', Energy and buildings, 86, 794-802 (2015)

10. S. Royer, S. Thil, T. Talbert, M. Polit, Energy and Buildings, 78, 231-237 (2014)

11. M. Lebied, F. Sick, Z. Choulli, A. El Bouardi, Case Studies in Thermal Engineering, 11, 125-134 (2018) doi:10.1016/j.csite.2018.01.007

12. M. E. L. Najjarine, A. G. Cheikh, Senkov: System analysis and applied information science, 1, 31-34 (2015)

13. D. Popescu, I. Borza, 5th International Conference on Smart Cities and Green ICT Systems (SMARTGREENS),456-461 (2016) doi:10.1007/978-3-319-63712-9

14. N. A. Tseligorov, E. N. Tseligorova, G. M. Mafura, Computational Problems in Science and Engineering, 75-80 (2014) doi:10.1007/978-3-319-15765-8

15. F. Gazdoš, P. Dostál, International journal of mathematical models and methods in applied sciences, 5, 1189-1196(2011)

16. R. Matušů, International journal of mathematical models and methods in applied sciences, 8, 95-102(2014)

17. N. A. Tseligorov, E. N. Tseligorova, G. M. Mafura, International Multi-Conference on Engineering, Computer and Information Sciences (SIBIRCON), 356-359 (2017) doi: 10.1109/SIBIRCON.2017.8109905.

18. R. Matušů, R. Prokop, Proceeding of the 15th WSEAS International Conference on Mathematical and Computational Methods in Science and Engineering, 8, 95-102 (2013) 